\title{
Vortex pairing in an unstable anticyclonic shear flow: discrete subharmonics of one pendulum day
}

\author{
By PIERRE FLAMENT ${ }^{1,2}$, RICK LUMPKIN ${ }^{1,3}$, \\ JEAN TOURNADRE AND LAURENCE ARMI I \\ ${ }^{1}$ Departement d'Oceanographie Spatiale, Institut Francais de Recherche pour l'Exploitation de \\ la Mer, 29280 Plouzane, France \\ ${ }^{2}$ Department of Oceanography, School of Ocean and Earth Science and Technology, \\ University of Hawai'i, Honolulu, HI 96822-2336, USA \\ ${ }^{3}$ Department of Oceanography, Florida State University, Tallahassee, FL 32306-4320, USA \\ ${ }^{4}$ Institute of Geophysics and Planetary Physics, Scripps Institution of Oceanography, \\ University of California San Diego, La Jolla, CA 92093-0225, USA
}

(Received 23 March 2001 and in revised form 28 April 2001)

Observations of the downstream evolution of an oceanic zonal horizontal shear flow at a Reynolds number of about $10^{11}$, formed as the westward North Equatorial Current passes the island of Hawaili, reveal finite-amplitude anticyclonic vortices resulting from instability of the shear. The initial orbital period of the vortices is exactly one pendulum day (3.1 days at this latitude), centrifugal instability presumably inhibiting stronger vortices from forming. As they move downstream, they appear to pair and merge into successively larger vortices, in a geometric sequence of longer orbital periods; three subharmonic transitions are suggested with final orbital periods near 6, 12 and 24 days.

\section{Introduction}

The observations reported here were made in a nearly ideal geophysical laboratory. As the westward branch of the North Pacific wind-driven circulation - the North Equatorial Current - reaches the southernmost point of the island of Hawaici $\left(19^{\circ} \mathrm{N}\right.$ $155.7^{\circ} \mathrm{W}$; figure 1), it separates into a detached shear layer which is unstable, forming vigorous anticyclonic vortices, recurrently observed west of the island (Wyrkti 1982; Mitchum 1995).

Seventy satellite-tracked drifting buoys drogued at a depth of $15 \mathrm{~m}$ (Niller et al. 1995) were deployed between 1994 and 1996 to study the formation, downstream evolution and dynamics of this shear layer. Additional buoys deployed off California and near the Equator eventually reached the area, augmenting the data set to a total of 286 buoy-years (Lumpkin \& Flament 2001). Measurements of sea surface height anomaly above the mean geoid were available from the ERS-1 and TOPEX/POSEIDON satellite altimeters, with respective repeat cycles of 35 and 10 days, and longitudinal resolutions of 80 and $270 \mathrm{~km}$ (LeTraon et al. 1995). Altimetric surface height anomaly maps provide views of the geostrophic stream function of the flow (Stewart 1985, Chapter 14). 


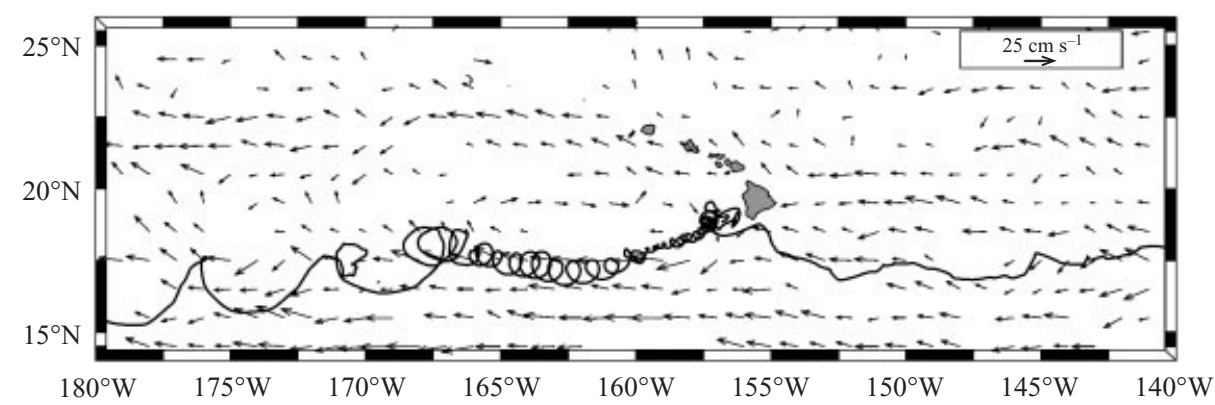

FIGURE 1. Mean currents around Hawai'i derived from all drifting buoys having sampled the area. The buoy trajectory discussed in $\S 2$ is superimposed.

Our observations suggest that vortex merging is an important mechanism for the downstream growth of this geophysical-scale shear layer. At a Reynolds number of about $10^{11}$, it shares many characteristics with free shear layers observed at moderate Reynolds numbers in the laboratory (Browand \& Winant 1973; Winant \& Browand 1974; Browand \& Roshko 1974), but, being subject to an external scale set by the baroclinic deformation radius, has a distinctly different growth rate.

\section{A buoy trajectory}

Most buoys encountered strong vortices west of the islands. A remarkable trajectory is shown in figure 2(a). For more than 11 months in 1994, this buoy remained trapped in an anticyclonic (clockwise) flow, drifting westward from $156^{\circ} \mathrm{W}$ to $180^{\circ} \mathrm{W}$ at an average speed of $11 \mathrm{~cm} \mathrm{~s}^{-1}$.

A time series of the orbital period of the buoy around the centre of the vortex is shown in figure $2(b)$. The periods were obtained by least-square fitting one-wavelength cycloids to successive loops of the trajectory. The orbital period had rapid transitions near $160^{\circ} \mathrm{W}$ (day 160 ), $166^{\circ} \mathrm{W}$ (day 234) and $168^{\circ} \mathrm{W}$ (day 274). Between them, the period remained relatively stable with medians of 2.9 days, 6.0 days, 12.6 days and 24.9 days, suggesting period doubling at each transition.

This vortex is clearly seen in the altimetric height, sampled along the path of the centre of the cycloids. Surface height anomaly has a $30 \mathrm{~cm}$ amplitude maximum propagating westward (figure $2 c$ ). The path of the vortex centre, inferred from the cycloid fits, corresponds precisely to the ridge of surface height anomaly. Vortex merging is suggested by the altimetry: a second ridge of height anomaly, trailing the main vortex by about $3^{\circ}$ longitude, eventually coalesces with it near $168^{\circ} \mathrm{W}$, around day 275 , precisely when the third period doubling occurs. A weaker leading vortex appears to coalesce with it near $166^{\circ} \mathrm{W}$, around day 240 , about the time of the second period doubling. A sketch of the paths of the main vortex, and of various leading and trailing vortices, is shown in figure $2(d)$.

The radius of the core of the vortex, estimated by the half-width of Gaussians least-square fitted to each altimetric profile, grew at each transition (figure $2 e$ ); the orbiting radius was always smaller than the altimetric radius, suggesting that the buoy remained trapped in the core of the vortex at all times, and that the transitions to longer periods were not the result of the buoy progressively escaping the vortex. The discontinuous nature of the orbital period is striking when visualized in the speed versus radius plot of individual loops (figure 3 ): the periods cluster in the vicinity of $3,6,12$ and 24 days. 
(a)

(b)

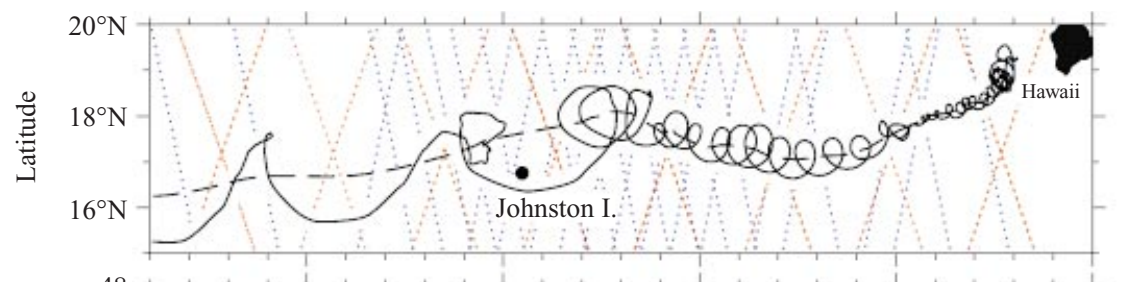

(c)
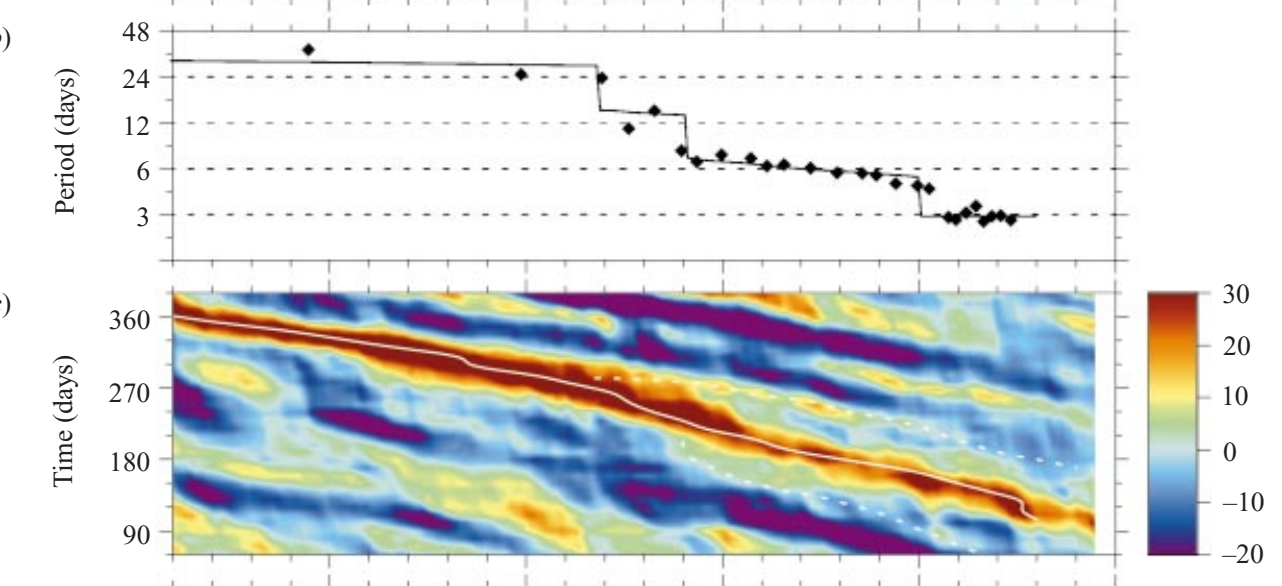

(d)

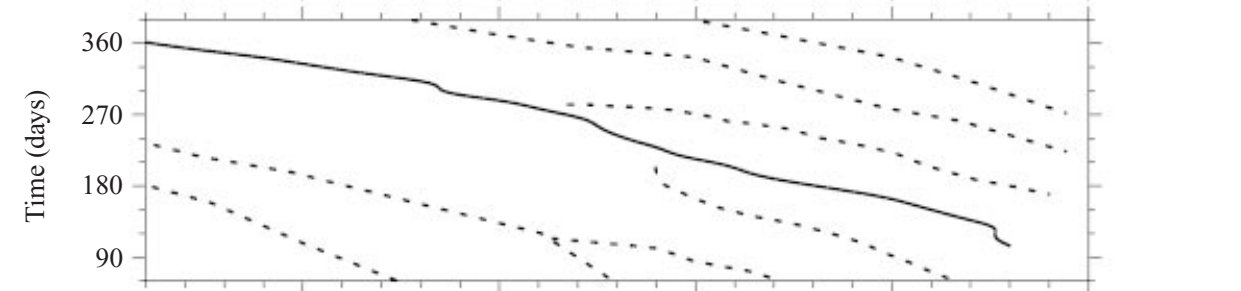

(e)

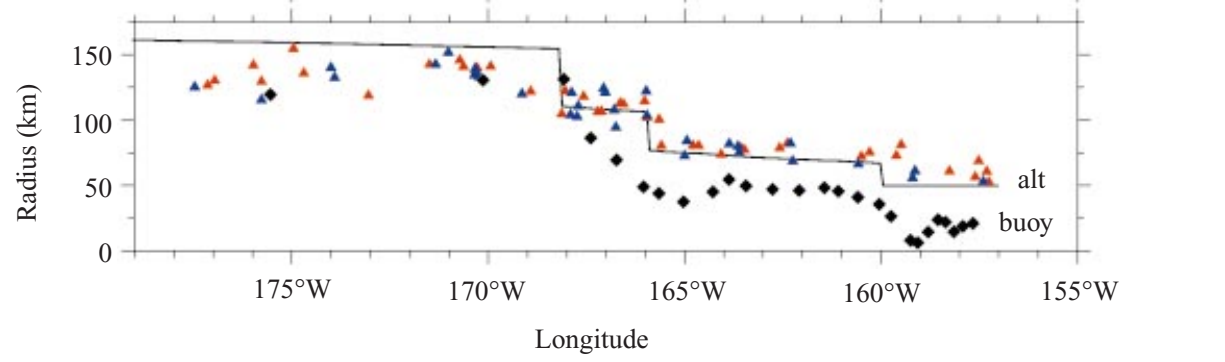

Figure 2. (a) Trajectory of the drifting buoy between April and December 1994. The path of the centre of the vortex is shown by a dashed line. The repeat tracks of the ERS and TOPEX satellite altimeters are shown by dotted lines. (b) Orbital period of the buoy around the centre of the vortex $\left(\log _{2}\right.$ scale). (c) Sea surface height in $\mathrm{cm}$ from the altimeters, sampled along the path of the centre, as a function of longitude and time in days of 1994. (d) Sketch of the propagation of the main vortex (solid line), and of various leading and trailing vortices (dashed lines). (e) Radius of the vortex inferred from the altimetry (triangles), and from the orbit of the buoy (diamonds). The radius and orbital period hindcasted by the simple model discussed in $\S 4$ are overlaid on $(b)$ and $(e)$.

Cycloids result from the superposition of a rotation and a translation. Four intervals between the successive orbital period transitions were chosen, between days 91 and 160, between days 160 and 234, between days 234 and 274, and between days 274 and 360 , to follow the vortex in the frame of reference translating with its centre, obtained from the individual cycloidal fits.

Surface height anomaly was mapped in this translating frame of reference, relative 


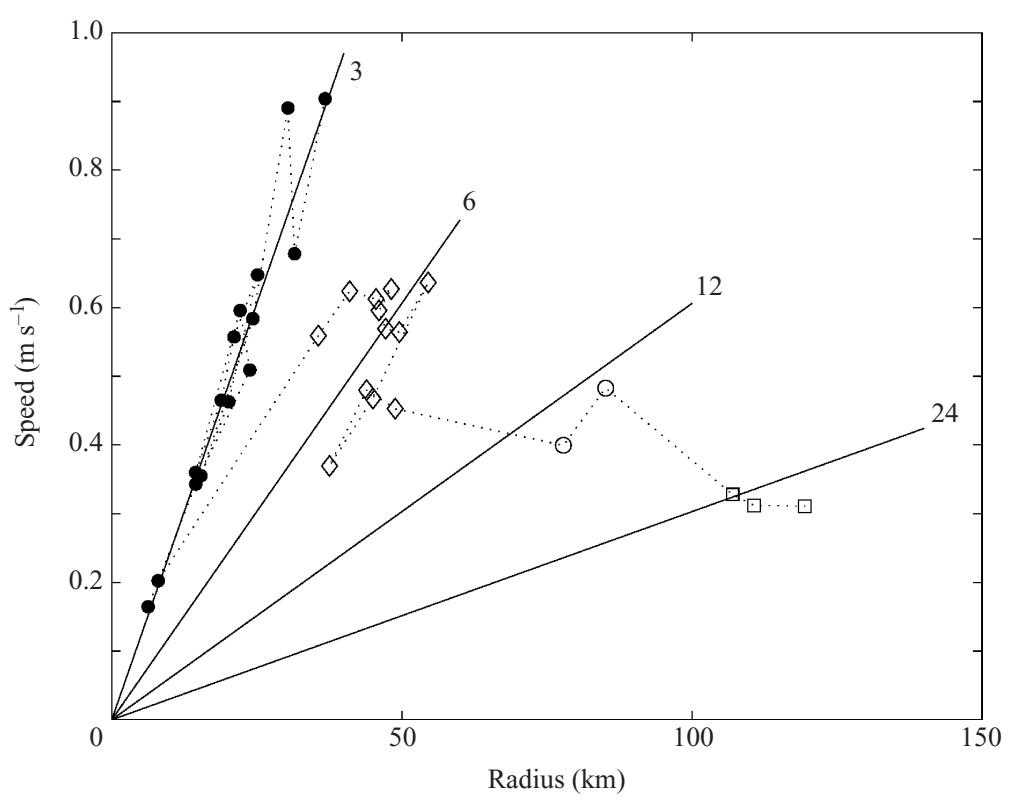

FIGURE 3. Azimuthal speed of the buoy versus distance from the centre. Each individual cycloidal loop corresponds to a symbol. The symbols are linked sequentially by a dashed line to show the chronology of the transitions, with the origin near the number 3 . The slopes corresponding to orbital periods of 2, 4, 8 and 16 times the local inertial period are drawn.

to which the flow can be considered approximately steady (Pingree 1996; Flament et al. 1996; Kennan \& Flament 2000). For each interval, the surface height profiles in the moving coordinates are remarkably consistent, despite their large spatio-temporal separation in fixed coordinates, and reveal coherent anticyclonic vortices (figure $4 a$ ). When viewed in these translating coordinates, the buoy trajectory becomes nearly circular closed loops (figure $4 b$ ). The azimuthally averaged surface height (figure $4 c$ ), and azimuthal velocity computed assuming cyclo-geostrophy (figure $4 d$ ), indicate that the vortices had an anticyclonic inner core in solid-body rotation, where speed grew linearly with radial distance, and a radially sheared outer layer of cyclonic vorticity.

The motion of the vortices as they propagate westward, and eventually merge, is also seen in successive maps of surface height anomaly (figure 5). Each map is estimated by gridding the data available in each 10-day period along the altimeter tracks shown, and therefore has variable spatial resolution and sampling noise. In spite of this, the vortices are clearly identified. The main vortex is first observed at $158^{\circ} \mathrm{W}$ during the $140 / 149$ period. The trailing vortex appeared near the island during the 198/208 period. It then followed a more northern but faster path than the main vortex. Around day 280, the two vortices became engulfed into a single, albeit noisy, region of high surface height, and can no longer be distinguished from each other. Possible mergings of smaller vortices closer to the island are buried in the noise and cannot be observed. A new vortex is also observed to form near the islands during the $239 / 249$ period.

\section{A statistical view}

While this buoy trajectory was chosen to reveal the transitions of orbital period, it is by no means unique: many buoys looped in the vicinity of $18^{\circ} \mathrm{N}$, with initial 
orbital frequencies around 3 days maintained for several orbits, and at least four others displayed similar frequency transitions. In particular, a buoy which circled an anticyclone from September 1996 to January 1997, changed suddenly from a 3- to 6-day orbital period (Lumpkin 1998).

To establish the generality of such orbital periods, multiples of one pendulum day, the entire set of drifting buoys having sampled the area $15^{\circ}$ to $19^{\circ} \mathrm{N}$ and $155^{\circ}$ to $180^{\circ} \mathrm{W}$ between 1982 and 1997 , were examined statistically. The buoy discussed in $\S 2$ was excluded from this set to strengthen the argument. The average Lagrangian rotary velocity spectra, estimated from all 120-day buoy trajectory segments, are shown in figure 6. The error bars were obtained through stochastic simulations (Efron \& Gong 1983): the spectra were repeatedly estimated by randomly drawing, with repetition and omission, among the set of 96 independent trajectory segments, and the standard errors were computed from these repeated realizations.

Large, significant peaks are seen in the clockwise anticyclonic spectrum, at exactly 6 and 12 days, with a smaller peak at 3 days. In contrast, there is much less energy in the counterclockwise cyclonic spectrum (the peak at 5 days in the cyclonic spectrum corresponds to cyclones generated occasionally further north through an entirely different wind-driven process). Spectra east of the islands, or south of $16^{\circ} \mathrm{N}$, display no such peaks, and have little or no rotary asymmetry (Lumpkin \& Flament 2001).

\section{Discussion}

The largest negative relative vorticity that a barotropic anticyclonic vortex can sustain at latitude $\lambda$ is $-f=-2 \Omega \sin \lambda$, where $\Omega$ is the angular frequency of the Earth's rotation. Equivalently, the minimum achievable Rossby number for a stable vortex is $R o=-1$. Stronger anticyclones would be destroyed by centrifugal instability, and re-form at $R o$ in the stable range (Rayleigh 1916; Kloosterziel \& van Heijst 1991; Carnevale et al. 1997). In the laboratory, anticyclonic shear flows in a homogeneous rotating fluid have also been found to be destabilized when $R o<-1$ (Bidokhti \& Tritton 1992; Tritton 1992). A similar vorticity limit can be anticipated for stratified baroclinic geophysical shear layers (D'Asaro 1988). This limit corresponds to an angular frequency of $\omega=-f / 2$, or a period of 3.1 days at the latitude of the separation point, remarkably similar to the period of the first set of loops. Such 'half-inertial' (Chew \& Bushnell 1990) vortices with a period of one pendulum day have also been observed at lower latitude (Flament et al. 1996; Kennan \& Flament 2000) and in the arctic (D'Asaro 1988).

These observations suggest that the unstable shear layer, initially very narrow as it detached from the island, rapidly grew to a width precisely set by the centrifugal stability condition, and then consisted of half-inertial vortices for as many as 16 orbital periods. Although the details of the initial growth and instability were not captured by the buoy, a shipboard Acoustic Doppler Current Profiler section, taken $12 \mathrm{~km}$ downstream from the separation point in August 1994, revealed horizontal vorticity near $-4.5 \times 10^{-5} \mathrm{~s}^{-1}(-0.95 f)$, to more than $100 \mathrm{~m}$ depth (Lempkin 1998). Growth to the centrifugal stability limit would then have occurred over less than 14 hours of advection - much less than a pendulum day.

The subharmonic steps in rotation rates are consistent with vortex merging. When two identical vortices, idealized as cylinders spinning at the same rate, merge into a single cylinder of the same height, the radius increases by $\sqrt{2}$ and the rotational period doubles, if angular momentum and mass are conserved; slightly smaller increases occur when more realistic baroclinic eddies merge (Lumpkin et al. 2000). 


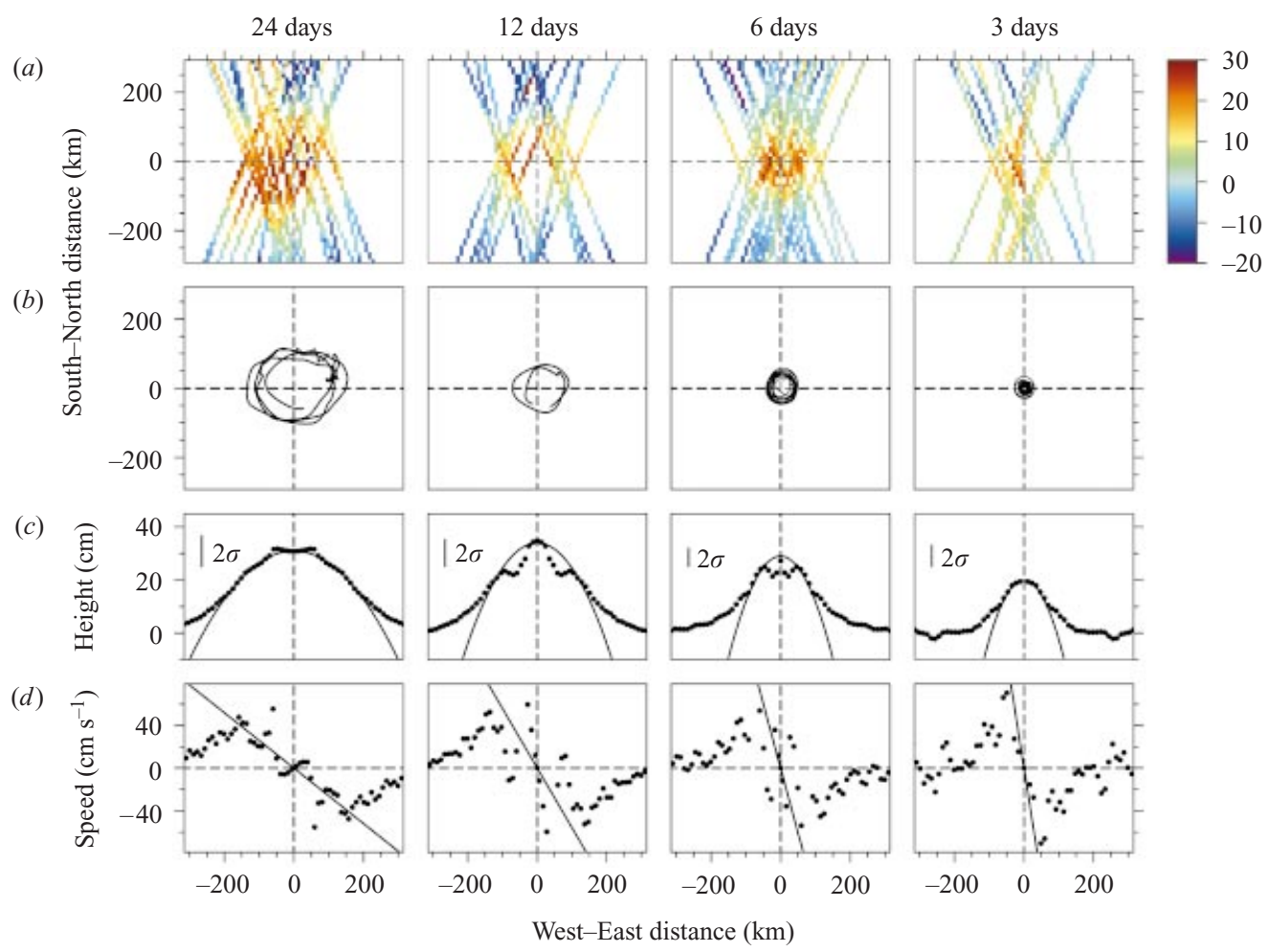

FiguRE 4. Fields viewed in the frame of reference translating with the centre, for each interval between orbital period transitions. Plotted right to left: $\approx 3$ days, day 91 to day $160 ; \approx 6$ days, until day $234 ; \approx 12$ days, until day 274 , and $\approx 24$ days, until day 414 (18 February 1995). (a) Sea surface height for the satellite altimeter tracks crossing the vortex during each time interval, in $\mathrm{cm}$. (b) Relative trajectory of the drifting buoy. (c) Azimuthally averaged surface height from the altimeters as a function of distance from the centre, in $\mathrm{cm}$ (dots). The 2-standard-deviation bars are shown for each profile. The parabolas correspond to solid-body rotation at the buoy's median orbital period. $(d)$ Azimuthal velocity computed from the surface height, assuming cyclo-geostrophy, in $\mathrm{cm} \mathrm{s}^{-1}$ (dots). Straight lines correspond to solid-body rotation.

A simple diagnostic model, combining entrainment and vortex merging at the times of the observed orbital period steps (Lumpkin 1998), accounts well for the evolution of the present vortices (figure $2 b, e$ ). The baroclinic vortices are idealized as a solid-body anticyclonic core, surrounded by an outer shell of constant cyclonic vorticity, bringing the velocity to zero at the periphery. They are confined to an active upper layer over an infinite quiescent lower layer ( $1 \frac{1}{2}$-layer approximation), and are in cyclogeostrophic balance (i.e. the nonlinear terms are retained). At each merging with a presumably identical vortex, conservation of the mass of the cores and of total energy and total angular momentum are required, and entrainment of surrounding

FigURE 5. Ten-day-average maps of sea surface height anomaly derived from the satellite altimeters. The end/start days of each averaging period are indicated to the left of the maps. The path of the centre of the main vortex, inferred from the buoy trajectory, is shown in white. The path of the centre of the trailing vortex, inferred from separate Gaussian fits to the altimetric heights, is shown in black (upper dashed line in figure $2 c$ ). The segments of the paths corresponding to the respective averaging periods are thickened. The satellite tracks used to construct each map are shown by thin black lines. The colour scale gives surface height in $\mathrm{cm}$. 
Vortex pairing in an anticyclonic shear flow
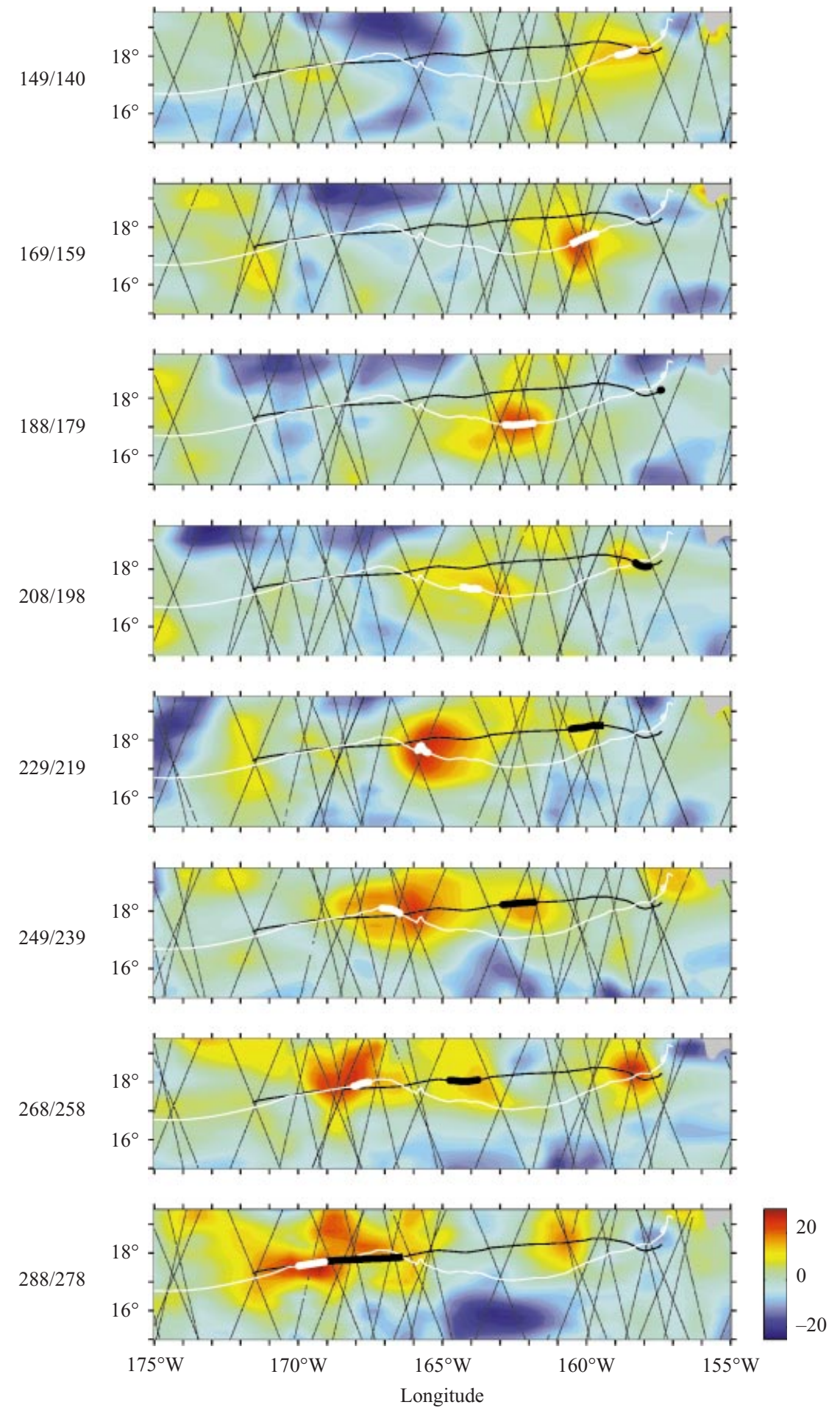

FIGURE 5. For caption see facing page. 


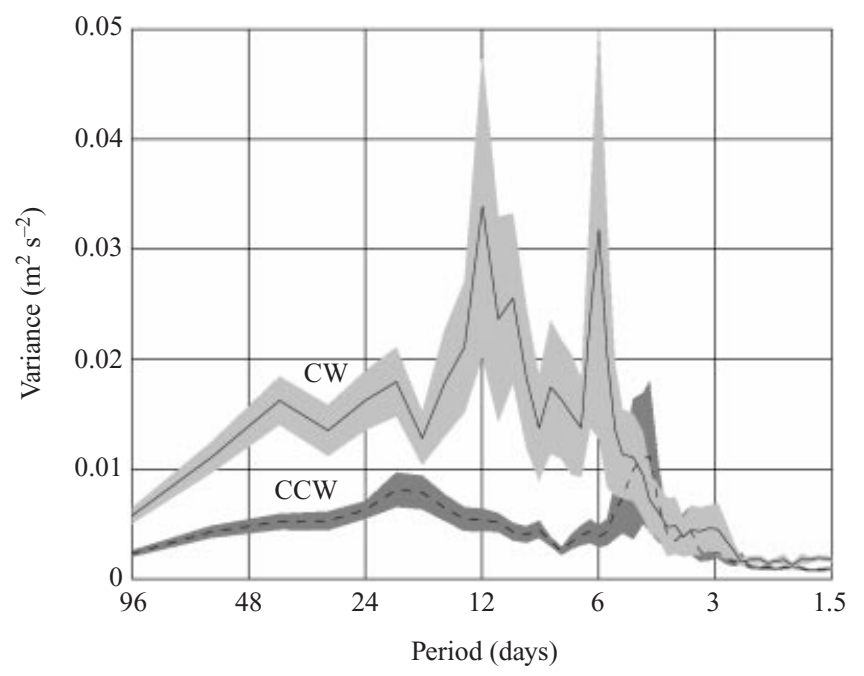

FIGURE 6. Lagrangian rotary velocity spectra estimated from 120-day buoy trajectory segments, excluding the buoy shown in figure 1 , covering the area $15^{\circ}$ to $19^{\circ} \mathrm{N}, 155^{\circ}$ to $180^{\circ} \mathrm{W}$. The clockwise (anticyclonic) spectrum is shown in light grey and the counterclockwise (cyclonic) spectrum in dark grey. The error bars were obtained by stochastic simulations over 96 independent segments.

fluid and filament shedding are neglected (Lumpkin et al. 2000). Between merging events, vortices entrain surrounding fluid at a rate proportional to their perimeter, and to the shear across the outer shell, with a proportionality constant $A_{H}=0.6 \mathrm{~m}^{2} \mathrm{~s}^{-1}$ tuned to best fit the observed spin-down. For a reduced gravity $g \Delta \rho / \rho=0.035 \mathrm{~m} \mathrm{~s}^{-2}$, an upper layer thickness of $230 \mathrm{~m}$, an initial period of one pendulum-day, and initial core and outer shell radii of $50 \mathrm{~km}$ and $150 \mathrm{~km}$, this simple model hindcasts the evolutions of period and radius well.

The observations suggest that the cascade of eddy energy to larger scales proceeded through vortex merging and period doubling. A 'quantization' of the orbital frequencies occurred, at ideally $f / 2$ (3 days), $f / 4$ (6 days), $f / 8(12$ days), $f / 16$ (24 days), the memory of the initial period being progressively lost as vortices entrained surrounding fluid. Slight departures from these canonical frequencies are attributed to the baroclinic structure of the vortices (Lumpkin et al. 2000), and to asymmetric mergings.

Such geophysical shear layers share many characteristics with free shear layers which are self-similar at high Reynolds numbers. Although here the Reynolds number, based on molecular viscosity, is about $10^{11}$, the equivalent Reynolds number, based on typical turbulent viscosity of the ocean surface mixed layer (Flament \& Armi 2000), would be $5 \times 10^{4}$. Simple dimensional analysis and laboratory experiments show that the width of free shear layers $\delta$ grows linearly with downstream distance $l$ as $\delta / l \approx 0.2$ (Brown \& Roshko 1974). In contrast, geophysical shear layers would be subject to an external dimensional scale imposed by the Earth's rotation, gravity and stratification through the internal radius of deformation $R_{R o}=\left(g^{\prime} h\right)^{1 / 2} / f$, with possibly a second external dimensional scale set by $\beta$, the variation of planetary vorticity with latitude, resulting in a nonlinear growth law of the form $\delta \propto l \phi\left(\delta / R_{R o}\right)$, where $\phi()$ is a function as yet unknown. From our observations, growth rate and hence entrainment are about half that for non-rotating shear layers, with $\delta / l \approx 0.11$. 
J. Firing and M. Pazos assisted with data collection and processing. This work was supported by the Office of Naval Research, the National Atmospheric and Oceanographic Administration, Joint Institute for Marine and Atmospheric Research, and our host institutions. SOEST contribution number 5484, JIMAR contribution number $01-338$.

\section{REFERENCES}

Bidokhti, A. A. \& Tritton, D. J. 1992 The structure of a turbulent free shear layer in a rotating fluid. J. Fluid Mech. 241, 469-502.

BrowAND, F. K. \& WinANT, C. D. 1973 Laboratory observations of shear-layer instability in a stratified fluid. Boundary Layer Met. 5, 67-77.

Brown, G. L. \& Roshko, A. 1974 On density effects and large structure in turbulent mixing layer. J. Fluid Mech. 64, 775-816.

Carnevale, G. F., Briscolini, M., Kloosterziel, R. C. \& Vallis, G. K. 1997 Three-dimensionally perturbed vortex tubes in a rotating flow. J. Fluid Mech. 341, 127-163.

Chew, F. \& Bushnell, M. H. 1990 The half-inertial flow in the eastern equatorial Pacific: a case study. J. Phys. Oceanogr. 20, 1124-1133.

D’Asaro, E. A. 1988 Generation of submesoscale vortices: A new mechanism. J. Geophys. Res. 93, 6685-6693.

Efron, B. \& GonG, G. 1983 A leisurely look at the bootstrap, the jacknife and the cross-validation. The American Statistician 37, 36-48.

Flament, P. \& Armi, L. 2000 The shear, convergence, thermohaline finestructure of a front. J. Phys. Oceanogr. 30, 51-66.

Flament, P., Kennan, S. C., Knox, R., Niller, P. \& Bernstein, R. 1996 The three-dimensional structure of an upper ocean vortex in the tropical Pacific. Nature 382, 610-613.

Kennan, S. C. \& Flament, P. J. 2000 Observations of a tropical instability vortex. J. Phys. Oceanogr. 30, 2277-2301.

Kloosterziel, R. C. \& van Heisst, G. J. F. 1991 An experimental study of unstable barotropic vortices in a rotating fluid. J. Fluid Mech. 223, 1-24.

LeTraon, P. Y., Gaspar, P., Bouyssel, F. \& Makhmara, H. 1995 Using TOPEX/Poseidon data to enhance ERS-1 orbit. J. Atmos. Oceanogr. Tech. 12, 161-170.

LumpKIN, C. 1998 Eddies and currents of the Hawaiian Islands. PhD thesis, School of Ocean and Earth Sciences and Technology, University of Hawai'i at Manoa.

Lumpkin, R. \& Flament, P. 2001 Lagrangian statistics in the central North Pacific. J. Mar. Syst. 29, 141-155.

Lumpkin, R., Flament, P., Kloosterziel, R. \& Armi, L. 2000 Vortex merging in a $1 \frac{1}{2}$-layer fluid on an $f$-plane. J. Phys. Oceanogr. 30, 233-242.

Mitchum, G. T. 1995 The source of 90-day oscillations at Wake Island. J. Geophys. Res. 100, 2459-2475.

Nitler, P. P., Sybrandy, A. S., Bi, K., Poulain, P. M. \& Bitterman, D. 1995 Measurements of the water-following capability of holey-sock and TRISTAR drifters. Deep-Sea Res. 42, 1951-1964.

PingreE, R. D. 1996 A shallow subtropical subducting westward propagating eddy. Phil. Trans. R. Soc. Lond. 354, 979-1026.

RaYleigh, LORD 1916 On the dynamics of revolving fluids. Proc. R. Soc. Lond. A 93, 148-154.

Stewart, R. H. 1985 Methods of Satellite Oceanography. University of California Press.

TRITTON, D. J. 1992 Stabilization and destabilization of structure of turbulent shear flow in a rotating fluid. J. Fluid Mech. 241, 503-523.

Winant, C. D. \& Browand, F. K. 1974 Vortex pairing: the mechanism of turbulent mixing layer growth at moderate Reynolds numbers. J. Fluid Mech. 63, 237-255.

WyrTKI, K. 1982 Eddies in the Pacific North Equatorial Current. J. Phys. Oceanogr. 12, 746-749. 